\title{
Complex mass renormalization in EFT
}

\section{Djukanovic}

Institut für Kernphysik, Johannes Gutenberg-Universität, J.J. Becherweg 45, D-55099 Mainz, Germany

E-mail: dalibor@kph.uni-mainz.de

\section{J. Gegelia*}

Institut für Kernphysik, Johannes Gutenberg-Universität, J.J. Becherweg 45, D-55099 Mainz, Germany

and

High Energy Physics Institute of TSU, Tbilisi, Georgia

E-mail: gegelia@kph.uni-mainz.de

\section{A. Keller}

Institut für Kernphysik, Johannes Gutenberg-Universität, J.J. Becherweg 45, D-55099 Mainz, Germany

E-mail: kellerakph.uni-mainz.de

\section{S. Scherer}

Institut für Kernphysik, Johannes Gutenberg-Universität, J.J. Becherweg 45, D-55099 Mainz, Germany

E-mail: scherer@kph.uni-mainz.de

We consider an effective field theory of unstable particles (resonances) using the complex-mass renormalization. As an application we calculate the masses and the widths of the $\rho$ meson and the Roper resonance.

6th International Workshop on Chiral Dynamics, CD09

July 6-10, 2009

Bern, Switzerland

\footnotetext{
* Speaker.
} 


\section{Introduction}

The construction of consistent chiral effective field theories with heavy degrees of freedom is a non-trivial problem. For example, in baryon chiral perturbation theory the usual power counting is violated if one uses the dimensional regularization and the minimal subtraction scheme [1]. The current solutions to this problem either involve the heavy-baryon approach [2] or use a suitably chosen renormalization condition $[3,4,5,6]$. Due to the small mass difference between the nucleon and the $\Delta(1232)$ in comparison with the nucleon mass, the $\Delta$ resonance can be consistently included in the framework of effective field theory $[7,8,9,10,11]$.

On the other hand, the treatment of the $\rho$ meson or the inclusion of heavier baryon resonances such as the Roper resonance is more complicated. We address the issue of power counting in such effective theories by using the complex-mass renormalization scheme [12, 13, 14], which can be understood as an extension of the on-mass-shell renormalization scheme to unstable particles.

As an application we consider the masses and the widths of the $\rho$ meson and the Roper resonance. More details can be found in Refs. $[15,16]$.

\section{Rho meson}

We start with the most general effective Lagrangian for $\rho$ and $\omega$ mesons and pions in the parametrization of the model III of Ref. [17]:

$$
\mathscr{L}=\mathscr{L}_{\pi}^{(2)}+\mathscr{L}_{\rho \pi}+\mathscr{L}_{\omega}+\mathscr{L}_{\omega \rho \pi}+\cdots
$$

The individual expressions relevant for the calculations of this work read

$$
\begin{aligned}
\mathscr{L}_{\pi}^{(2)} & =\frac{F^{2}}{4} \operatorname{Tr}\left[\partial_{\mu} U\left(\partial^{\mu} U\right)^{\dagger}\right]+\frac{F^{2} M^{2}}{4} \operatorname{Tr}\left(U^{\dagger}+U\right), \\
\mathscr{L}_{\rho \pi} & =-\frac{1}{2} \operatorname{Tr}\left(\rho_{\mu \nu} \rho^{\mu v}\right)+\left[M_{\rho}^{2}+\frac{c_{x} M^{2} \operatorname{Tr}\left(U^{\dagger}+U\right)}{4}\right] \operatorname{Tr}\left[\left(\rho^{\mu}-\frac{i \Gamma^{\mu}}{g}\right)\left(\rho_{\mu}-\frac{i \Gamma_{\mu}}{g}\right)\right], \\
\mathscr{L}_{\omega} & =-\frac{1}{4}\left(\partial_{\mu} \omega_{v}-\partial_{v} \omega_{\mu}\right)\left(\partial^{\mu} \omega^{v}-\partial^{v} \omega^{\mu}\right)+\frac{M_{\omega}^{2} \omega_{\mu} \omega^{\mu}}{2}, \\
\mathscr{L}_{\omega \rho \pi} & =\frac{1}{2} g_{\omega \rho \pi} \varepsilon_{\mu v \alpha \beta} \omega^{v} \operatorname{Tr}\left(\rho^{\alpha \beta} u^{\mu}\right),
\end{aligned}
$$

where

$$
\begin{aligned}
U & =u^{2}=\exp \left(\frac{i \vec{\tau} \cdot \vec{\pi}}{F}\right), \quad \rho^{\mu}=\frac{\vec{\tau} \cdot \vec{\rho}^{\mu}}{2}, \\
\rho^{\mu v} & =\partial^{\mu} \rho^{v}-\partial^{v} \rho^{\mu}-i g\left[\rho^{\mu}, \rho^{v}\right], \\
\Gamma_{\mu} & =\frac{1}{2}\left[u^{\dagger} \partial_{\mu} u+u \partial_{\mu} u^{\dagger} u_{\mu}=i\left[u^{\dagger} \partial_{\mu} u-u \partial_{\mu} u^{\dagger}\right] .\right.
\end{aligned}
$$

All the fields and parameters in Eqs. (2.1) are bare quantities. In order to increase the readability of the expressions we have omitted the usual subscript 0 . In Eqs. (2.1), $F$ denotes the pion-decay constant in the chiral limit, $M^{2}$ is the lowest-order expression for the squared pion mass, $M_{\rho}$ and 
$M_{\omega}$ refer to the bare $\rho$ and $\omega$ masses, $g, c_{x}$, and $g_{\omega \rho \pi}$ are coupling constants. We use the KSFR relation $[18,19]$

$$
M_{\rho}^{2}=2 g^{2} F^{2} .
$$

To perform the renormalization we express the bare quantities in terms of renormalized ones:

$$
\begin{aligned}
M_{\rho, 0} & =M_{R}+\delta M_{R}, \\
c_{x, 0} & =c_{x}+\delta c_{x}, \\
& \ldots
\end{aligned}
$$

We apply the complex-mass renormalization scheme $[12,13,14]$ and choose $M_{R}^{2}=\left(M_{\chi}-i \Gamma_{\chi} / 2\right)^{2}$ as the pole of the $\rho$-meson propagator in the chiral limit. $M_{\chi}$ and $\Gamma_{\chi}$ are the pole mass and the width of the $\rho$ meson in the chiral limit, respectively. Both are input parameters in our approach. In the complex-mass renormalization scheme, the counterterms are in general complex quantities.

The presence of large external momenta of the $\rho$ meson leads to a considerable complication in the power counting for loop diagrams. It is necessary to investigate all possible flows of the external momenta through the internal lines of a loop diagram. Next, one needs to determine the chiral orders for all flows of external momenta. Finally, the smallest of these orders is defined as the chiral order of the given diagram.

The power counting rules are as follows. Let $q$ collectively stand for a small quantity such as the pion mass. A pion propagator counts as $\mathscr{O}\left(q^{-2}\right)$ if it does not carry large external momenta and as $\mathscr{O}\left(q^{0}\right)$ if it does. A vector-meson propagator counts as $\mathscr{O}\left(q^{0}\right)$ if it does not carry large external momenta and as $\mathscr{O}\left(q^{-1}\right)$ if it does. The pion mass counts as $\mathscr{O}\left(q^{1}\right)$, the vector-meson mass as $\mathscr{O}\left(q^{0}\right)$, and the width as $\mathscr{O}\left(q^{1}\right)$. Vertices generated by the effective Lagrangian of Goldstone bosons $\mathscr{L}_{\pi}^{(n)}$ count as $\mathscr{O}\left(q^{n}\right)$. Derivatives acting on heavy vector mesons count as $\mathscr{O}\left(q^{0}\right)$. The contributions of vector meson loops can be absorbed systematically in the parameters of the effective Lagrangian.

The dressed propagator, expressed in terms of the self energy

$$
i \Pi_{\mu v}^{a b}(p)=i \delta^{a b}\left[g_{\mu \nu} \Pi_{1}\left(p^{2}\right)+p_{\mu} p_{\nu} \Pi_{2}\left(p^{2}\right)\right]
$$

has the form

$$
i S_{\mu \nu}^{a b}(p)=-i \delta^{a b} \frac{g_{\mu \nu}-p_{\mu} p_{v} \frac{1+\Pi_{2}\left(p^{2}\right)}{M_{R}^{2}+\Pi_{1}\left(p^{2}\right)+p^{2} \Pi_{2}\left(p^{2}\right)}}{p^{2}-M_{R}^{2}-\Pi_{1}\left(p^{2}\right)+i 0^{+}} .
$$

The pole of the propagator is found as the (complex) solution to the following equation:

$$
z-M_{R}^{2}-\Pi_{1}(z)=0 .
$$

We define the pole mass and the width of the $\rho$ meson by parameterizing

$$
z=\left(M_{\rho}-i \Gamma / 2\right)^{2} .
$$

The solution to Eq. (2.7) can be found perturbatively as a loop expansion

$$
z=z^{(0)}+z^{(1)}+z^{(2)}+\cdots
$$




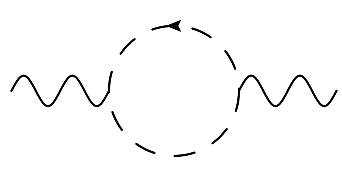

(a)

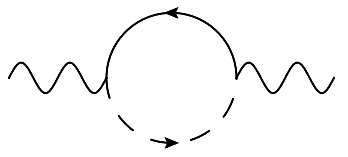

(b)

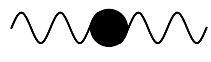

(c)

Figure 1: One-loop contributions to the $\rho$-meson self-energy at $\mathscr{O}\left(q^{3}\right)$. The dashed, solid, and wiggly lines correspond to the pion, the $\omega$ meson, and the $\rho$ meson, respectively.

Each of these terms has its own chiral expansion. Up to third chiral order the pole reads

$$
z=z^{(0)}=M_{R}^{2}+c_{x} M^{2}
$$

The one-loop contributions to the vector self-energy up to $\mathscr{O}\left(q^{3}\right)$ are shown in Fig. 1. The contributions of diagrams (a) and (b) to $\Pi_{1}$ are given by

$$
\begin{aligned}
\Pi_{1(a)}= & \frac{g^{2}\left[2 A_{0}\left(M^{2}\right)-\left(p^{2}-4 M^{2}\right) B_{0}\left(p^{2}, M^{2}, M^{2}\right)\right]}{16 \pi^{2}(n-1)}, \\
\Pi_{1(b)}= & -\frac{(n-2) g_{\omega \rho \pi}^{2}}{64 \pi^{2}(n-1)}\left\{M^{4} B_{0}\left(p^{2}, M^{2}, M_{\omega}^{2}\right)-\left[2 B_{0}\left(p^{2}, M^{2}, M_{\omega}^{2}\right) M_{\omega}^{2}+A_{0}\left(M^{2}\right)-A_{0}\left(M_{\omega}^{2}\right)\right.\right. \\
& \left.+2 B_{0}\left(p^{2}, M^{2}, M_{\omega}^{2}\right) p^{2}\right] M^{2}+B_{0}\left(p^{2}, M^{2}, M_{\omega}^{2}\right) p^{2}+M_{\omega}^{2}\left[B_{0}\left(p^{2}, M^{2}, M_{\omega}^{2}\right) M_{\omega}^{2}\right. \\
& \left.\left.+A_{0}\left(M^{2}\right)-A_{0}\left(M_{\omega}^{2}\right)\right]-\left[2 B_{0}\left(p^{2}, M^{2}, M_{\omega}^{2}\right) M_{\omega}^{2}+A_{0}\left(M^{2}\right)+A_{0}\left(M_{\omega}^{2}\right)\right] p^{2}\right\}
\end{aligned}
$$

Using dimensional regularization with $n$ space-time dimensions, the loop functions read

$$
\begin{aligned}
A_{0}\left(m^{2}\right)= & -32 \pi^{2} \lambda m^{2}-2 m^{2} \ln \frac{m}{\mu} \\
B_{0}\left(p^{2}, m_{1}^{2}, m_{2}^{2}\right)= & -32 \pi^{2} \lambda+2 \ln \frac{\mu}{m_{2}}-1 \\
& -\frac{1}{2}\left(1+\frac{m_{2}^{2}}{m_{1}^{2}(\omega-1)}\right){ }_{2} F_{1}\left(1,2 ; 3 ; 1+\frac{m_{2}^{2}}{m_{1}^{2}(\omega-1)}\right)-\frac{\omega}{2}{ }_{2} F_{1}(1,2 ; 3 ; \omega), \\
\omega= & \frac{m_{1}^{2}-m_{2}^{2}+p^{2}+\sqrt{\left(m_{1}^{2}-m_{2}^{2}+p^{2}\right)^{2}-4 m_{1}^{2} p^{2}}}{2 m_{1}^{2}}
\end{aligned}
$$

where ${ }_{2} F_{1}(a, b ; c ; z)$ is the standard hypergeometric function, $\mu$ is the scale parameter and

$$
\lambda=\frac{1}{16 \pi^{2}}\left\{\frac{1}{n-4}-\frac{1}{2}\left[\ln (4 \pi)+\Gamma^{\prime}(1)+1\right]\right\} .
$$

The $\rho \pi \pi$ vertex in diagram (a) should count as $\mathscr{O}\left(q^{0}\right)$. However, its large component does not contribute to $\Pi_{1}$. Therefore, the $\Pi_{1}$ part of diagram (a) has order $\mathscr{O}\left(q^{4}\right)$. Diagram (c) contains the contributions of the counterterms.

In diagram (b) we take $M_{\omega}=M_{R}$. 
We fix the counterterms such that the pole in the chiral limit stays at $M_{R}^{2}$. The contributions of diagrams (a), (b), and (c) to the pole, expanded up to $\mathscr{O}\left(q^{4}\right)$, read

$$
z^{(1)}=\frac{g^{2} M^{4}}{16 \pi^{2} M_{R}^{2}}\left(3-2 \ln \frac{M^{2}}{M_{\chi}^{2}}-2 i \pi\right)-\frac{g_{\omega \rho \pi}^{2} M^{3} M_{\chi}}{24 \pi}-\frac{g_{\omega \rho \pi}^{2} M^{4}\left(\ln \frac{M^{2}}{M_{\chi}^{2}}-1\right)}{32 \pi^{2}}+\frac{i g_{\omega \rho \pi}^{2} M^{3} \Gamma_{\chi}}{48 \pi} .
$$

\section{Roper Resonance}

The most general effective Lagrangian, relevant for the subsequent calculation of the pole of the Roper propagator at order $\mathscr{O}\left(q^{3}\right)$ reads:

$$
\mathscr{L}=\mathscr{L}_{0}+\mathscr{L}_{\pi}^{(2)}+\mathscr{L}_{R}+\mathscr{L}_{N R}+\mathscr{L}_{\Delta R}
$$

where $\mathscr{L}_{0}$ is given by

$$
\begin{aligned}
\mathscr{L}_{0}= & \bar{N}\left(i \not \partial-m_{N 0}\right) N+\bar{R}\left(i \not \partial-m_{R 0}\right) R \\
& -\bar{\Psi}_{\mu} \xi^{\frac{3}{2}}\left[\left(i \not \partial-m_{\Delta 0}\right) g^{\mu \nu}-i\left(\gamma^{\mu} \partial^{v}+\gamma^{v} \partial^{\mu}\right)+i \gamma^{\mu} \not \partial \gamma^{v}+m_{\Delta 0} \gamma^{\mu} \gamma^{v}\right] \xi^{\frac{3}{2}} \Psi_{v} .
\end{aligned}
$$

Here, $N$ and $R$ denote nucleon and Roper isospin doublets with bare masses $m_{N 0}$ and $m_{R 0}$, respectively. $\Psi_{v}$ are the vector-spinor isovector-isospinor Rarita-Schwinger fields of the $\Delta$ resonance [20] with bare mass $m_{\Delta 0}$ and $\xi^{\frac{3}{2}}$ is the isospin-3/2 projector (see Ref. [10] for more details).

The interaction terms $\mathscr{L}_{R}, \mathscr{L}_{N R}$, and $\mathscr{L}_{\Delta R}$ are constructed following Ref. [21]. At leading order

$$
\mathscr{L}_{R}^{(1)}=\frac{g_{R}}{2} \bar{R} \gamma^{\mu} \gamma_{5} u_{\mu} R .
$$

The next-to-leading-order Roper Lagrangian is given by

$$
\mathscr{L}_{R}^{(2)}=c_{1,0}^{*}\left\langle\chi_{+}\right\rangle \bar{R} R
$$

where $c_{1,0}^{*}$ is a coupling constant and $\chi_{+}=M^{2}\left(U+U^{\dagger}\right)$. The nucleon-Roper interaction reads

$$
\mathscr{L}_{N R}^{(1)}=\frac{g_{N R}}{2} \bar{R} \gamma^{\mu} \gamma_{5} u_{\mu} N+\text { h.c. }
$$

Finally, the leading-order interaction between the delta and the Roper is given by

$$
\mathscr{L}_{\Delta R}^{(1)}=-g_{\Delta R} \bar{\Psi}_{\mu} \xi^{\frac{3}{2}}\left(g^{\mu v}+\tilde{z} \gamma^{\mu} \gamma^{v}\right) u_{v} R+\text { h.c. },
$$

where we take the "off-mass-shell parameter" $\tilde{z}=-1$.

To renormalize the loop diagrams, we apply the complex-mass renormalization and write:

$$
\begin{aligned}
m_{R 0} & =z_{\chi}+\delta z_{\chi}, \\
m_{N 0} & =m+\delta m, \\
m_{\Delta 0} & =z_{\Delta \chi}+\delta z_{\Delta \chi}, \\
c_{1,0}^{*} & =c_{1}^{*}+\delta c_{1}^{*}, \\
\cdots &
\end{aligned}
$$




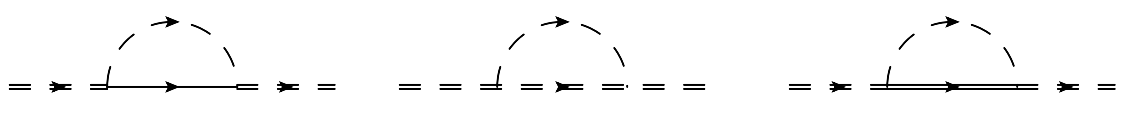

(a)

(b)

(c)

Figure 2: One-loop self-energy diagrams of the Roper. The dashed, solid, double-dashed, and double-solid lines correspond to the pion, nucleon, Roper, and delta, respectively.

where $z_{\chi}$ is the complex pole of the Roper propagator in the chiral limit, $m$ is the mass of the nucleon in the chiral limit, and $z_{\Delta \chi}$ is the pole of the delta propagator in the chiral limit.

We organize our perturbative calculation by applying the standard power counting of Refs. [22], i.e., an interaction vertex obtained from an $\mathscr{O}\left(q^{n}\right)$ Lagrangian counts as order $q^{n}$, a pion propagator as order $q^{-2}$, a nucleon propagator as order $q^{-1}$, and the integration of a loop as order $q^{4}$. In addition, we assign the order $q^{-1}$ to the $\Delta$ propagator and to the Roper propagator (carrying loop momenta). Within the complex-mass renormalization scheme, such a power counting is respected in the range of energies close to the Roper mass.

The dressed propagator of the Roper

$$
i S_{R}(p)=\frac{i}{\not p-z_{\chi}-\Sigma_{R}(\not p)},
$$

where $\Sigma_{R}(\not p)$ denotes the self-energy, has a complex pole which is obtained from the equation

$$
z-z_{\chi}-\Sigma_{R}(z)=0
$$

To order $\mathscr{O}\left(q^{3}\right)$ the Roper self-energy consists of a tree-order contribution

$$
\Sigma_{\text {tree }}=4 c_{1}^{*} M^{2},
$$

and the loop diagrams shown in Fig. 2. For the diagrams (a), (b), and (c) of Fig. 2 we obtain

$$
\begin{aligned}
& \Sigma_{(a)}=\frac{3 g_{N R}^{2}}{128 \pi^{2} F^{2}}\left[\hat{O}_{1}(m) A_{0}\left(m^{2}\right)+\hat{O}_{2}(m) A_{0}\left(M^{2}\right)+\hat{O}_{3}(m) B_{0}\left(p^{2}, m^{2}, M^{2}\right)\right], \\
& \Sigma_{(b)}=\frac{3 g_{R}^{2}}{128 \pi^{2} F^{2}}\left[\hat{O}_{1}\left(z_{\chi}\right) A_{0}\left(z_{\chi}^{2}\right)+\hat{O}_{2}\left(z_{\chi}\right) A_{0}\left(M^{2}\right)+\hat{O}_{3}\left(z_{\chi}\right) B_{0}\left(p^{2}, z_{\chi}^{2}, M^{2}\right)\right], \\
& \Sigma_{(c)}=\frac{g_{\Delta R}^{2}}{48 \pi^{2} F^{2}}\left[\hat{O}_{4}+\hat{O}_{5} A_{0}\left(z_{\Delta \chi}^{2}\right)+\hat{O}_{6} A_{0}\left(M^{2}\right)+\hat{O}_{7} B_{0}\left(p^{2}, z_{\Delta \chi}^{2}, M^{2}\right)\right],
\end{aligned}
$$

where

$$
\begin{aligned}
& \hat{O}_{1}(x)=\not p\left(1+\frac{x^{2}}{p^{2}}\right)+2 x \\
& \hat{O}_{2}(x)=\not p\left(1-\frac{x^{2}}{p^{2}}\right), \\
& \hat{O}_{3}(x)=\not p\left[-p^{2}\left(1-\frac{x^{2}}{p^{2}}\right)^{2}+M^{2}\left(1+\frac{x^{2}}{p^{2}}\right)\right]+2 M^{2} x .
\end{aligned}
$$




$$
\begin{aligned}
& \hat{O}_{4}=\frac{1}{6}\left[3 \not p z_{\Delta \chi}^{2}-12 p^{2} z_{\Delta \chi}-4 \not p p^{2}+4 p^{2} \frac{p^{2}-3 M^{2}}{z_{\Delta \chi}}+\not p \frac{2\left(p^{2}\right)^{2}-3 M^{4}-8 p^{2} M^{2}}{z_{\Delta \chi}^{2}}\right], \\
& \hat{O}_{5}=\frac{1}{p^{2}}\left[\not p z_{\Delta \chi}^{2}+2 p^{2} z_{\Delta \chi}-\not p\left(2 M^{2}+p^{2}\right)+2 p^{2} \frac{p^{2}-M^{2}}{z_{\Delta \chi}}+\not p \frac{\left(M^{2}-p^{2}\right)^{2}}{z_{\Delta \chi}^{2}}\right] \\
& \hat{O}_{6}=-\frac{1}{p^{2}}\left[\not p z_{\Delta \chi}^{2}+2 p^{2} z_{\Delta \chi}-2 M^{2} \not p-2 p^{2} \frac{M^{2}+p^{2}}{z_{\Delta \chi}}+\not p \frac{M^{4}-3 p^{2} M^{2}-\left(p^{2}\right)^{2}}{z_{\Delta \chi}^{2}}\right] \\
& \hat{O}_{7}=-\frac{1}{p^{2}}\left[\not p z_{\Delta \chi}^{2}+2 p^{2} z_{\Delta \chi}+\not p\left(p^{2}-M^{2}\right)\right]\left[z_{\Delta \chi}^{2}-2\left(M^{2}+p^{2}\right)+\frac{\left(M^{2}-p^{2}\right)^{2}}{z_{\Delta \chi}^{2}}\right]
\end{aligned}
$$

To implement the complex-mass renormalization scheme, in analogy to Ref. [6], we expand the self-energy loop diagrams in powers of $M, \not p-z_{\chi}$, and $p^{2}-z_{\chi}^{2}$, which all count as $\mathscr{O}(q)$. We subtract those terms which violate the power counting. The subtraction terms at $\not p=z_{\chi}$ read

$$
\begin{aligned}
\Sigma_{(a)}^{\mathrm{ST}}= & -\frac{3 g_{N R}^{2}\left(m+z_{\chi}\right)^{2}}{128 \pi^{2} F^{2} z_{\chi}}\left[\left(m-z_{\chi}\right)^{2} B_{0}\left(z_{\chi}^{2}, 0, m^{2}\right)-A_{0}\left(m^{2}\right)\right] \\
& +\frac{3 g_{N R}^{2}\left(m+z_{\chi}\right) M^{2}}{64 \pi^{2} F^{2} z_{\chi}^{3}}\left[-2 m^{3} \ln \frac{m}{\mu}-i \pi m^{3}+z_{\chi}^{2} m-32 \pi^{2} z_{\chi}^{3} \lambda\right. \\
& \left.+\left(m^{3}-z_{\chi}^{3}\right) \ln \frac{z_{\chi}^{2}-m^{2}}{\mu^{2}}+i \pi z_{\chi}^{3}\right] \\
\Sigma_{(b)}^{\mathrm{ST}}= & \frac{3 g_{R}^{2} z_{\chi}}{32 \pi^{2} F^{2}} A_{0}\left(z_{\chi}^{2}\right)-\frac{3 g_{R}^{2} z_{\chi} M^{2}}{32 \pi^{2} F^{2}}\left[32 \pi^{2} \lambda+2 \ln \frac{z_{\chi}}{\mu}-1\right] \\
\Sigma_{(c)}^{\mathrm{ST}}= & -\frac{g_{\Delta R}^{2}}{288 F^{2} \pi^{2} z_{\Delta \chi}^{2} z_{\chi}}\left[6\left(z_{\Delta \chi}-z_{\chi}\right)^{2}\left(z_{\Delta \chi}+z_{\chi}\right)^{4} B_{0}\left(z_{\chi}^{2}, 0, z_{\Delta \chi}^{2}\right)\right. \\
& +z_{\chi}^{2}\left(-3 z_{\Delta \chi}^{4}+12 z_{\chi} z_{\Delta \chi}^{3}+4 z_{\chi}^{2} z_{\Delta \chi}^{2}-4 z_{\chi}^{3} z_{\Delta \chi}-2 z_{\chi}^{4}\right) \\
& \left.-6\left(z_{\Delta \chi}^{4}+2 z_{\chi} z_{\Delta \chi}^{3}-z_{\chi}^{2} z_{\Delta \chi}^{2}+2 z_{\chi}^{3} z_{\Delta \chi}+z_{\chi}^{4}\right) A_{0}\left(z_{\Delta \chi}^{2}\right)\right] \\
& +\frac{g_{\Delta R}^{2} M^{2}}{72 \pi^{2} F^{2} z_{\Delta \chi}^{2} z_{\chi}^{3}}\left[-6 i \pi z_{\Delta \chi}^{6}-6\left(2 z_{\Delta \chi}+3 z_{\chi}\right) z_{\Delta \chi}^{5} \ln \frac{z_{\Delta \chi}}{\mu}-9 i \pi z_{\chi} z_{\Delta \chi}^{5}+6 z_{\chi}^{2} z_{\Delta \chi}^{4}\right. \\
& +9 z_{\chi}^{3} z_{\Delta \chi}^{3}+3 z_{\chi}^{4} z_{\Delta \chi}^{2}-288 \pi^{2} \lambda z_{\chi}^{5} z_{\Delta \chi}+9 i \pi z_{\chi}^{5} z_{\Delta \chi}+z_{\chi}^{6}-192 \pi^{2} \lambda z_{\chi}^{6} \\
& \left.+\left(6 z_{\Delta \chi}^{6}+9 z_{\chi} z_{\Delta \chi}^{5}-9 z_{\chi}^{5} z_{\Delta \chi}-6 z_{\chi}^{6}\right) \ln \frac{z_{\chi}^{2}-z_{\Delta \chi}^{2}}{\mu^{2}}+6 i \pi z_{\chi}^{6}\right]
\end{aligned}
$$

The above expressions of Eq. (3.14) are exactly canceled by contributions of $\delta z_{\chi}$ and $\delta c_{1}^{*}$. The pole of the Roper propagator to third order is given by the expression

$$
z=z_{\chi}-4 c_{1}^{*} M^{2}+\left[\Sigma_{(a)}+\Sigma_{(b)}+\Sigma_{(c)}\right]_{\not p=z_{\chi}}-\Sigma_{(a)}^{\mathrm{ST}}-\Sigma_{(b)}^{\mathrm{ST}}-\Sigma_{(c)}^{\mathrm{ST}} .
$$

It is easily shown that the expansion of Eq. (3.15) satisfies the power counting, i.e. is of $\mathscr{O}\left(q^{3}\right)$.

\section{Summary}

We have considered an effective field theory of resonances interacting with Goldstone bosons using the complex-mass renormalization scheme. A systematic power counting emerging within 
this scheme allows one to calculate the physical quantities in powers of small parameters. As an application we have calculated the pole masses and the widths of the $\rho$ meson and the Roper resonance which are of particular interest in the context of lattice extrapolations. The masses and the widths in the chiral limit are considered as input parameters within this approach.

\section{Acknowledgments}

This work was supported by the Deutsche Forschungsgemeinschaft (SFB 443). We thank the organizers for a very pleasant meeting.

\section{References}

[1] J. Gasser, M. E. Sainio and A. Švarc, Nucl. Phys. B307, 779 (1988).

[2] E. E. Jenkins and A. V. Manohar, Phys. Lett. B 255, 558 (1991).

[3] H. B. Tang, arXiv:hep-ph/9607436.

[4] T. Becher and H. Leutwyler, Eur. Phys. J. C 9, 643 (1999).

[5] J. Gegelia and G. Japaridze, Phys. Rev. D 60, 114038 (1999).

[6] T. Fuchs, J. Gegelia, G. Japaridze, and S. Scherer, Phys. Rev. D 68, 056005 (2003).

[7] T. R. Hemmert, B. R. Holstein, and J. Kambor, J. Phys. G 24, 1831 (1998).

[8] V. Pascalutsa and D. R. Phillips, Phys. Rev. C 67, 055202 (2003).

[9] V. Bernard, T. R. Hemmert, and U. -G. Meißner, Phys. Lett. B 565, 137 (2003).

[10] C. Hacker, N. Wies, J. Gegelia, and S. Scherer, Phys. Rev. C 72, 055203 (2005).

[11] V. Pascalutsa, M. Vanderhaeghen, and S. N. Yang, Phys. Rept. 437, 125 (2007).

[12] R. G. Stuart, in $Z^{0}$ Physics, ed. J. Tran Thanh Van (Editions Frontieres, Gif-sur-Yvette, 1990), p.41.

[13] A. Denner, S. Dittmaier, M. Roth, and D. Wackeroth, Nucl. Phys. B560, 33 (1999).

[14] A. Denner and S. Dittmaier, Nucl. Phys. Proc. Suppl. 160, 22 (2006).

[15] D. Djukanovic, J. Gegelia, A. Keller, and S. Scherer, Phys. Lett. B 680, 235 (2009).

[16] D. Djukanovic, J. Gegelia, and S. Scherer, arXiv:0903.0736 [hep-ph].

[17] G. Ecker, J. Gasser, H. Leutwyler, A. Pich, and E. de Rafael, Phys. Lett. B 223, 425 (1989).

[18] K. Kawarabayashi and M. Suzuki, Phys. Rev. Lett. 16, 255 (1966).

[19] Riazuddin and Fayyazuddin, Phys. Rev. 147, 1071 (1966).

[20] W. Rarita and J. S. Schwinger, Phys. Rev. 60, 61 (1941).

[21] B. Borasoy, P. C. Bruns, U.-G. Meißner, and R. Lewis, Phys. Lett. B 641, 294 (2006).

[22] S. Weinberg, Nucl. Phys. B363, 3 (1991). 